\title{
Driving Sleep Slow Oscillations by Auditory Closed-Loop Stimulation-A Self-Limiting Process
}

\author{
Hong-Viet V. Ngo, ${ }^{1,2,3 *}$ Arjan Miedema, ${ }^{1,4 \star}$ Isabel Faude, ${ }^{1}$ Thomas Martinetz, ${ }^{2}$ Matthias Mölle, ${ }^{1,5}$ and Jan Born ${ }^{1,6}$ \\ ${ }^{1}$ Institute for Medical Psychology and Behavioral Neurobiology, University of Tuebingen, 72076 Tuebingen, Germany, ${ }^{2}$ Institute for Neuro- and \\ Bioinformatics and ${ }^{3}$ Graduate School for Computing in Medicine and Life Science, University of Luebeck, 23562 Luebeck, Germany, ${ }^{4}$ SILS-CNS, University \\ of Amsterdam, 1012 WX Amsterdam, The Netherlands, ${ }^{5}$ Center of Brain, Behavior and Metabolism, University of Luebeck, 23562 Luebeck, Germany, and \\ ${ }^{6}$ Center for Integrative Neuroscience, University of Tuebingen, 72076 Tuebingen, Germany
}

The $<1 \mathrm{~Hz}$ EEG slow oscillation (SO) is a hallmark of slow-wave sleep (SWS) and is critically involved in sleep-associated memory formation. Previous studies showed that SOs and associated memory function can be effectively enhanced by closed-loop auditory stimulation, when clicks are presented in synchrony with upcoming SO up states. However, increasing SOs and synchronized excitability also bear the risk of emerging seizure activity, suggesting the presence of mechanisms in the healthy brain that counter developing hypersynchronicity during SOs. Here, we aimed to test the limits of driving SOs through closed-loop auditory stimulation in healthy humans. Study I tested a "Driving stimulation" protocol (vs "Sham") in which trains of clicks were presented in synchrony with SO up states basically as long as an ongoing SO train was identified on-line. Study II compared Driving stimulation with a "2-Click" protocol where the maximum of stimuli delivered in a train was limited to two clicks. Stimulation was applied during SWS in the first $210 \mathrm{~min}$ of nocturnal sleep. Before and after sleep declarative word-pair memories were tested. Compared with the Sham control, Driving stimulation prolonged SO trains and enhanced SO amplitudes, phase-locked spindle activity, and overnight retention of word pairs (all ps $<0.05$ ). Importantly, effects of Driving stimulation did not exceed those of 2-Click stimulation $(p>0.180)$, indicating the presence of a mechanism preventing the development of hypersynchronicity during SO activity. Assessment of temporal dynamics revealed a rapidly fading phase-locked spindle activity during repetitive click stimulation, suggesting that spindle refractoriness contributes to this protective mechanism.

Key words: auditory stimulation; closed-loop control; declarative memory consolidation; fast spindles; sleep; slow oscillations

\section{Introduction}

The slow oscillation (SO) is the major EEG characteristic of slowwave sleep (SWS), with frequencies of $\sim 0.8 \mathrm{~Hz}$ in humans (Achermann and Borbély, 1997; Steriade, 2003). It reflects highly synchronized cortical activity, with down states representing widespread neuronal hyperpolarization and up states representing widespread depolarization and distinctly enhanced network excitability (Olcese et al., 2010; Timofeev, 2011). SOs originate from neocortical networks, preferentially involved in prior information encoding during wakefulness, possibly due to ongoing miniature excitatory postsynaptic potentials (Bazhenov et al., 2002; Huber et al., 2004). Functionally, they are thought to me-

\footnotetext{
Received July 29, 2014; revised Jan. 29, 2015; accepted Feb. 17, 2015.

Author contributions: H.-V.V.N., A.M., M.M., and J.B. designed research; H.-V.V.N., A.M., and I.F. performed research; H.-V.V.N., A.M., I.F., T.M., M.M., and J.B. analyzed data; H.-V.V.N., A.M., T.M., M.M., and J.B. wrote the paper.

This work was supported by the Deutsche Forschungsgemeinschaft (TR-SFB 654) and a BMBF Grant (01GQ0973).

*H.-V.V.N. and A.M. contributed equally to this work.

The authors declare no competing financial interests.

This article is freely available online through the J Neurosci Author Open Choice option.

Correspondence should be addressed to Jan Born, Institute for Medical Psychology and Behavioral Neuro-

biology, University of Tuebingen, Otfried-Mueller-Strasse 25, 72076 Tuebingen, Germany. E-mail: jan.born@uni-tuebingen.de.

DOI:10.1523/JNEUROSCI.3133-14.2015

Copyright $\odot 2015 \mathrm{Ngo}$, Miedema et al.

This is an Open Access article distributed under the terms of the Creative Commons Attribution License Creative Commons Attribution 4.0 International, which permits unrestricted use, distribution and reproduction in any medium provided that the original work is properly attributed.
}

diate a global renormalization of synapses potentiated during wakefulness (Tononi and Cirelli, 2014), though recent findings contradict this hypothesis (Chauvette et al., 2012; Seibt et al., 2012; Yang et al., 2014). Consistent evidence indicates that the SO promotes memory consolidation, especially of hippocampusdependent declarative memories (Marshall et al., 2006; Wilhelm et al., 2013). This function is presumably favored by thalamocortical spindles $(12-15 \mathrm{~Hz})$, which arise phase-locked to SO up states and support a redistribution of reactivated memory representations (Siapas and Wilson, 1998; Bergmann et al., 2012).

Beyond benefiting cognitive function, $\mathrm{SO}$ activity represents a state of increased likelihood of seizures. Hypersynchronicity, possibly facilitated by excess network resetting during SO down states, and reduced inhibitory signaling during $\mathrm{SO}$ up states, have been shown to favor epileptiform activity in hippocampal and neocortical networks (Nazer and Dickson, 2009; de Guzman et al., 2010; Chen et al., 2012). Conversely, these studies show that in the healthy brain mechanisms are at work during SOs that counter the development of hypersynchronicity, preventing the emergence of seizure-like activity, although the nature of these mechanisms is unclear.

The functional importance of SOs has stimulated approaches to enhance SOs using electrical and auditory stimulation procedures (Marshall et al., 2004; Massimini et al., 2007; Ngo et al., 2013a). Closed-loop stimulation revealed to be particularly effec- 


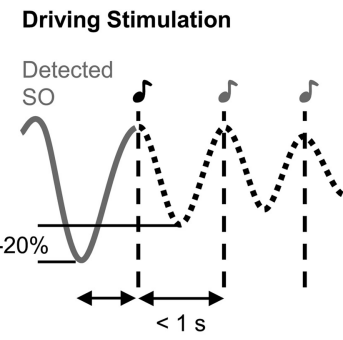

$\sim 0.5 \mathrm{~s}$

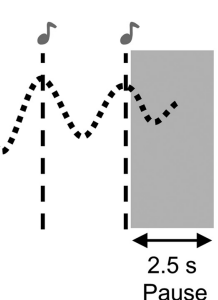

Pause
2-Click Stimulation

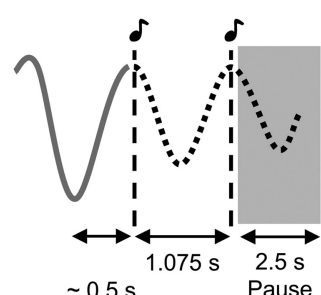

$\sim 0.5 \mathrm{~s}$

Pause
Figure 1. Stimulation protocols. Both the Driving stimulation (left) and the 2-Click stimulation protocols (right) relied on the identification of the negative half-wave peak of an $\mathrm{SO}$ in the ongoing EEG (prefiltered between 0.25 and $4 \mathrm{~Hz}$ ) recorded from AFz during SWS. Thus, an SO-negative peak was identified whenever the EEG signal crossed an initial threshold value of less than $-80 \mu \mathrm{V}$, which was followed by presenting a first click $\sim 0.5 \mathrm{~s}$ later. The initial threshold value was fine-tuned based on the largest negative amplitude during the preceding 5 s interval, but always $<-80 \mu \mathrm{V}$. The interval between detection of a negative SO peak and presentation of the first click was individually determined based on recordings in the adaptation night, such that the click occurred at the estimated succeeding positive SO peak. In the Driving stimulation protocol, presentation of the click was followed by the detection of a succeeding SO-negative peak within a 1 s post-click window. For each succeeding click, the threshold was lowered to $80 \%$ of the previous click. If the EEG signal did not cross this threshold within the $1 \mathrm{~s}$ post-click window, the detection algorithm paused for $2.5 \mathrm{~s}$. During the Sham condition SO detection was performed in an identical manner, however, without presentation of clicks. In the 2-Click stimulation protocol (adopted from $\mathrm{Ngo}$ et al., 2013b) presentation of the first click was always followed by presentation of a second click with a fixed interval of $1.075 \mathrm{~s}$. Then the algorithm paused for $2.5 \mathrm{~s}$.

tive in producing selective increases in SO activity, together with increases in phase-locked spindle activity and an improved consolidation of declarative word-pair memories (Ngo et al., 2013b). The closed-loop stimulation protocol of that study relied on the on-line identification of SO down states to time presentation of two clicks such that they fell into the predicted up states of two succeeding SO cycles. Because the on-line detection paused for $2.5 \mathrm{~s}$ following presentation of the two clicks, the protocol stimulated only shorter SO trains and was not apt to explore possible mechanisms that counter SO generation to prevent hypersynchronicity.

Here, we aimed at overdriving closed-loop SO stimulation in healthy humans with the primary goal to unravel mechanisms that counteract SO induction and possible hypersynchronicity, limiting its enhancing effects. To this end, we applied a closedloop "Driving stimulation" protocol in which trains of clicks were virtually continuously presented as long as an identifiable SO train was ongoing (Fig. 1). In Study I, Driving stimulation proved effective compared with a Sham control. However, in Study II directly comparing Driving stimulation with the "2Click" stimulation protocol described previously (Ngo et al., 2013b) Driving stimulation was not superior, despite its increased stimulatory drive.

\section{Materials and Methods}

Participants, experimental design, and procedures. In Study I, 18 subjects (mean age $23.8 \pm 0.6$ years, 10 men) participated; 16 other subjects ( $24.3 \pm$ 0.74 years, 6 men) participated in Study II. None of the subjects had participated in a similar experiment previously. All participants were healthy, free of medication, nonsmokers, and native German speakers. Routine examination ensured that they had no history of neurological or psychiatric disease, including any sleep disorder. Subjects had followed a regular sleep-wake rhythm for at least 4 weeks before the experiments. Before the first experimental night, they were accustomed to sleeping under laboratory conditions during an adaptation night, including the attachment of electrodes for EEG and polysomnographic recordings. On experimental days, subjects were required to get up at 7:00 A.M. and not to take any naps during the day. They were not allowed to consume alcohol or, after 3:00 P.M., caffeine-containing drinks. Written informed consent was obtained before the subject's participation. The experiments were approved by the ethics committee of the University of Tuebingen.

Both studies were performed according to a within-subject crossover design with each subject participating in two conditions (Study I: Driving stimulation vs Sham stimulation; Study II: Driving stimulation vs 2-Click stimulation). The order of conditions was balanced across subjects and both conditions for an individual were separated by an interval of at least $7 \mathrm{~d}$.

On experimental nights participants arrived at the sleep laboratory at 8:00 P.M. and were prepared for polysomnographic recordings. Between 9:00 and 10:30 P.M. they performed on the word-pair memory task and went to bed afterward. Polysomnographic recordings started at 11:00 P.M. (lights off). Auditory stimulation commenced $\sim 5$ min after the subject displayed stable sleep stage 2 (or deeper) for the first time after sleep onset, and was discontinued 210 min later. Stimulation was applied only when SWS was present. If SWS was ongoing at this time, stimulation was continued until the end of this SWS period. Subjects were awakened in the next morning after $7 \mathrm{~h}$ of sleep whenever they had entered light non-REM sleep, i.e., stages 1 or 2 . Approximately 30 min later, recall of word-pair memories was examined.

The word-pair memory task was adopted from a previous study (Ngo et al., 2013b) and comprised the successive presentation of 120 German word pairs. Cued recall was tested immediately after learning and again after sleep. Before and after sleep, tests also were applied to measure the subject's mood, tiredness, general alertness, vigilance, and executive functions (working memory and retrieval capabilities).

EEG recordings and polysomnography. The EEG was recorded continuously with a BrainAmp DC amplifier (Brain Products) from 19 channels (international 10-20 system: Fp1, Fp2, F7, F3, Fz, F4, F8, T3, C3, Cz, C4, T4, T5, P3, Pz, P4, T6, O1, O2) referenced to linked mastoids. Ag$\mathrm{AgCl}$ electrodes were used and impedances were always kept $<5 \mathrm{k} \Omega$. Signals were filtered between 0.03 and $150 \mathrm{~Hz}$, sampled at $500 \mathrm{~Hz}$, and stored for later off-line analysis on a PC together with the stimulation triggers. Vertical and horizontal eye movements (EOG) as well as EMG from electrodes attached to the chin were obtained for standard polysomnography and artifact detection.

Closed-loop auditory stimulation protocols. All stimulation protocols relied on the on-line detection of SO-negative half-wave peaks (i.e., down states) as described previously (Ngo et al., 2013b). For the detection procedure and stimulus delivery, an additional EEG recording system consisting of a D360 EEG amplifier (Digitimer) and a "Power1401 mk 2" high-performance data acquisition interface (Cambridge Electronic Design) connected to a separate PC were used. With this setup, the prefrontal EEG was recorded from an electrode at $\mathrm{AFz}$ (located on the midline centered between $\mathrm{Fpz}$ and $\mathrm{Fz}$ ) referenced to the average potential from linked electrodes attached to the earlobes. The EEG was filtered between 0.25 and $4 \mathrm{~Hz}$ and sampled at $200 \mathrm{~Hz}$. A custom-made script running under Spike2 software Version 7 (Cambridge Electronic Design) enabled response to the incoming EEG signal in real time. Based on the EEG signal obtained from $\mathrm{AFz}$, an auditory stimulus was triggered whenever the signal crossed an adaptive threshold toward larger negative values. On default the threshold was set to $-80 \mu \mathrm{V}$. Every $0.5 \mathrm{~s}$ it was updated to the minimal (i.e., largest negative) instantaneous EEG amplitude within the preceding $5 \mathrm{~s}$ interval, however, only if this value exceeded (in negativity) $-80 \mu \mathrm{V}$. This algorithm ensured a reliable and continuous detection of SOs of increasing and decreasing amplitude within an SWS period.

Auditory stimulation was applied only during SWS, and was halted whenever subjects showed arousals or REM sleep. In both stimulation protocols, upon detection of an SO-negative half-wave peak, the first stimulus was delivered after an individually adapted delay to ensure a temporal coincidence with the upcoming SO up state. The delay was determined based on the analysis of the average delay between the SOnegative and SO-positive peaks during the first SWS period of an individual's adaptation night (mean \pm SEM: $479.2 \pm 10.5 \mathrm{~ms}$ across all 34 subjects of both Study I and II). In the Driving stimulation protocol, further stimuli were applied when the EEG signal again met the threshold criterion within a $1 \mathrm{~s}$ time window starting with the preceding click presentation, whereby with each stimulus cycle the threshold value was 
decreased by $20 \%$ (with reference to the preceding threshold value) to alleviate repetitive stimulations. The $20 \%$ reduction rule was introduced based on pilot studies and observations indicating that, in the absence of stimulation, the amplitude of the negative SO peak gradually decreases across trains of several succeeding SO cycles. The Driving stimulation procedure resulted in an average interstimulus interval (ISI; between clicks of a train) of $973.0 \pm 11.7 \mathrm{~ms}$ with values ranging between $631.8 \pm$ 9.8 and $1484.4 \pm 13.9 \mathrm{~ms}$ (across subjects). In the 2-Click protocol, presentation of the second click followed after a fixed interval of $1.075 \mathrm{~s}$ (Ngo et al., 2013b). In both stimulation protocols the detection algorithm paused for $2.5 \mathrm{~s}$ after the last click applied. Exact timing of click presentations was marked in the EEG for later off-line analysis. In the Sham control condition of Study I, detections of SOs and marking of the EEG were performed as in the Driving stimulation condition. However, no stimuli were delivered. Note, inasmuch the algorithms for both 2-Click and Driving stimulation relied on constant time intervals (e.g., the individual delay between a detected SO-negative half-wave peak and click presentation) they do not account for the temporal jitter inherent to the SO rhythm and are, thus, not entirely precise as to the estimated peak of the SO up phase. Although such imprecision is expected to nonspecifically affect every click presentation in both protocols, it needs to be explored in future studies whether in-phase timing of stimuli might be optimized with different algorithms (Cox et al., 2014).

Auditory stimuli were clicks of pink $1 /$ f noise of 50 ms duration with a $5 \mathrm{~ms}$ rising and falling flank, respectively. Sound volume was calibrated to $55 \mathrm{~dB}$ SPL. Stimuli were presented binaurally via MDR-EX35 in-ear headphones (Sony Deutschland). When asked in the next morning, in Study I, four subjects, and in Study II, three subjects reported that they had noticed clicks during the Driving Stimulation protocol; three subjects noticed the stimulation during the 2-Click protocol.

Memory task and control tests. To assess declarative memory, a pairedassociate learning task was used that had proven sensitive to effects of sleep as described previously (Plihal and Born, 1997; Marshall et al., 2006). The task consists of the sequential presentation of 120 moderately semantically related pairs of German nouns (e.g., Airplane-Tomato juice, Brain-Consciousness, etc.) on a monitor for $4 \mathrm{~s}$, with an ISI of $1 \mathrm{~s}$. A different word list was used for each of the subject's two experimental sessions. At learning before sleep, presentation of the list was followed by a task of cued recall, i.e., the subject had to respond by naming the second word on presentation of the first (cue) word of each pair, whereby the stimulus words of the word list appeared on the screen in a different order than during the foregoing presentation. The subject had unlimited time to recall the appropriate response word. Immediately after word recall the correct paired associate was revealed on the screen for $1 \mathrm{~s}$. At retrieval testing in the morning following sleep, cue words were again displayed in a newly randomized order and the subject was required to recall the appropriate response words and no feedback was given. Overnight memory retention is represented by the difference in the number of recalled words between morning retrieval testing and evening immediate recall.

Before and after sleep, the subject's mood and tiredness was assessed using the Positive and Negative Affect Schedule and the Stanford Sleepiness Scale. To control for general alertness and vigilance, all subjects performed a vigilance task before learning and retrieval testing. In this task (lasting $5 \mathrm{~min}$ ) a counter appeared at the center of a computer screen every $2-10 \mathrm{~s}$ and the participant had to stop it as quickly as possible by pressing a button. Additionally, in the morning after sleep, to assess the general capability to retrieve information from long-term memory, a word fluency task was used that required the subject to write down, within 2 min periods, respectively, as many kinds of jobs or hobbies beginning with the letter M or P. For working memory assessment, the digit span test of the Wechsler Adult Intelligence Scale was used, which requires the subject to repeat lists of orally presented digits forward and backward.

Analyses of sleep and spectral power during SWS. Generally, analyses were performed with Spike2 (Cambridge Electronic Design) and Brain Vision Analyzer 2 (Brain Products). EEG data were preprocessed with a bandpass filter of $0.3-30 \mathrm{~Hz}$ and EMG data with a high-pass filter of $5 \mathrm{~Hz}$. Sleep stages were scored off-line visually for succeeding $30 \mathrm{~s}$ epochs according to standard criteria (Rechtschaffen and Kales, 1968) using the
EEG recordings from C3 and C4. Total sleep time (TST), time spent in different sleep stages (wake; sleep stages 1, 2, 3, and 4; SWS, i.e., the sum of sleep stages 3 and 4; REM sleep), and movement arousals were determined for the whole night, as well as for the $210 \mathrm{~min}$ stimulation period. Sleep onset, i.e., the first occurrence of sleep stage 1 followed by stage 2 sleep, was defined with reference to lights off. For the analysis of spectral power during SWS, FFT was applied on the EEG data using a Hanning window with 4096 data points $(\sim 8.2 \mathrm{~s})$ resulting in a frequency resolution of $\sim 0.122 \mathrm{~Hz}$. Spectra were averaged across all $8.2 \mathrm{~s}$ windows and subsequently smoothed with a three-point moving average.

Stimulation-induced activity. To examine the EEG response induced by auditory stimulation the original EEG signal $(0.3-30 \mathrm{~Hz})$ was averaged time-locked to the marked click presentations. Before averaging, for the Driving stimulation and Sham condition click stimulations and respective "Sham marks" were categorized according to the number of successively presented clicks within a train (i.e., one, two, three, or four clicks; $>4$ clicks in a train were never presented). Sham marks of the Sham condition referred to the exact time points where a click would have occurred when applying the stimulation algorithm of the Stimulation condition. So, like in the Stimulation condition, also in the Sham condition, due to the spontaneous SO dynamics, trains of Sham marks could occur. To account for the temporal jitter between successive clicks (and Sham marks) in a train, intervals between them were standardized to 100 bins, with the EEG values representing mean values from each bin. Then, the EEG signal was averaged time locked to the first click. The induced SO response to a click (or Sham mark) was evaluated at frontocentral electrode sites (F3, Fz, F4, C3, Cz, and C4) with reference to the negative peak amplitude $\sim 50$ bins post stimulus. (Peak-to-peak amplitude measures of induced SO activity revealed basically the same results, but will not be reported here.) Corresponding analyses were performed to evaluate induced spindle activity defined by the rms EEG signal filtered in the fast $(12-15 \mathrm{~Hz})$ spindle band. Topographical maps of induced spindle power (phase-locked to SO up states) were generated with reference to their maximum value by third-order spherical spline interpolation.

Analysis of off-line-detected SOs. Off-line detection of SOs was restricted to SWS (although results did not essentially change with inclusion of stage 2 sleep epochs) and was based on an algorithm described in detail previously (Mölle et al., 2002). This algorithm is based on a virtual channel representing the mean EEG signal recorded from F3, Fz, F4, C3, $\mathrm{Cz}, \mathrm{C} 4, \mathrm{P} 3, \mathrm{Pz}$, and P4. In brief, the EEG is first low-pass filtered at $30 \mathrm{~Hz}$ and downsampled to $100 \mathrm{~Hz}$. For the identification of large SOs a lowpass filter of $3.5 \mathrm{~Hz}$ is applied. Then negative and positive peak potentials are derived from all intervals between consecutive positive-to-negative zero crossings (i.e., one negative and one positive peak for every interval). Only intervals with durations of $0.833-2 \mathrm{~s}$ (corresponding to a frequency of $0.5-1.2 \mathrm{~Hz}$ ) are included. We then calculated the mean values of the negative and positive peak potentials across the subject's two conditions and marked those intervals as SO epochs where the negative peak amplitude was lower than 1.25 times the mean negative peak amplitude and where the amplitude difference (positive peak minus negative peak) was larger than 1.25 times the mean amplitude difference. Negative half-wave peaks were used to mark SO events, which were then used to calculate auto-event correlations in a $\pm 5 \mathrm{~s}$ window around the negative half-wave peak with a bin size of $0.05 \mathrm{~s}$. The resulting counts in each bin were divided by the absolute number of SOs to obtain rates of SO occurrence independent of the number of identified SOs. Statistical comparisons between stimulation conditions were performed on succeeding $250 \mathrm{~ms}$ intervals.

Statistical analyses. Data from four participants were discarded because of missing SWS (two subjects) during the adaptation night or prolonged awakenings during the stimulation nights (two subjects), resulting in sample sizes of $n=16$ and $n=14$ for Study I and II, respectively. Regarding EEG analyses, in each study data from one further subject had to be discarded due to technical problems, resulting in final sample sizes of $n=15$ and $n=13$. For statistical analyses SPSS software was used. All data are presented as mean \pm SEM. Analyses generally relied on repeated-measures ANOVA with the within-subject factors Stimulation condition (Study I: Driving vs Sham; Study II: Driving vs 2-Click) and, when indicated, Topography, representing the 19 different 
EEG recording sites. The Greenhouse-Geisser correction of degrees of freedom was applied where appropriate. For subsequent pairwise testing, Student's paired $t$ tests were used. A $p$ value $<0.05$ was considered significant.

\section{Results}

Immediate effects of closed-loop stimulation on EEG activity Study I compared the effects of the Driving stimulation protocol with a Sham stimulation control condition in which stimulation time points were marked but no stimulation was applied. To compare the EEG response to closed-loop stimulation during SWS between the conditions, stimulus trains in both conditions were first categorized according to the number of clicks presented in a train (corresponding to the number of successively identified SO-negative half-wave peaks), i.e., whether stimulation occurred as a single click or in a train of two, three, or four stimuli (Fig. 2A). Then, time intervals between clicks were normalized to enable comparisons of whole stimulation trains between conditions. The resulting EEG signal was then averaged time-locked to the first click of a train.

Compared with spontaneously occurring SOs during Sham, Driving stimulation prolonged SO trains, i.e., produced a greater number of SO trains with two or more succeeding SO events (Fig. $2 A$, left). Thus, the number of SO trains with two, three, and four SO events in a row averaged $118.7 \pm 17.8$ in the Driving stimulation condition versus $79.1 \pm 12.3$ in the Sham condition $(p=0.004)$. Concurrently, the number of singular SO events was decreased during Driving stimulation ( $144.0 \pm 18.5$ vs $260.4 \pm 32.0$, during Sham, $p<0.001$ ). The total number of off-line-identified SOs was comparable after Driving and Sham stimulation $(400.9 \pm 62.8$ vs $409.4 \pm$ $39.5, p=0.885$ ). The prolonging effect on SO trains of Driving stimulation was likewise evident when analyzing autocorrelations for off-line-determined SO events (Fig. $2 B$, top), which revealed a significant increase of $\mathrm{SO}$ events followed by one or two further $\mathrm{SO}$ events (i.e., trains of two and three SOs, $p<0.021$, compared with Sham).

In contrast, in Study II, comparing the effects of Driving stimulation with the 2-Click protocol, no significant differences were observed for the respective SO event autocorrelations $(p>0.329$; Fig. $2 B$, bottom), indicating that both protocols were similarly effective in inducing SO trains, despite the increased number of clicks presented successively in a row in the Driving stimulation protocol. Accordingly, the overall number (451.5 \pm 50.0 vs $503.1 \pm 55.4, p=0.372)$ and amplitude $(164.3 \pm 7.380$ vs $173.1 \pm 8.2 \mu \mathrm{V}, p=0.086)$ of off-line-detected SO events was also comparable between the two stimulation conditions. Although with the Driving stimulation protocol more stimuli were presented in a train, the total number of clicks presented was higher in the 2-Click protocol $(691.1 \pm 90.0$ vs $494.8 \pm 66.4, p=$ 0.020 ; Fig. $2 A$, right), which simply reflects the fact that with the latter protocol presentation of the second click was unconditioned and did not require prior identification of a negative SO half-wave.

Compared with Sham stimulation, Driving stimulation also distinctly increased SO amplitudes. The increase was observed regardless of whether SOs occurred singularly or in trains of several succeeding SOs $\left(F_{(1,14)}>6.176, p<0.026\right.$, for all comparisons, except for the last of the 3 -click trains: $F_{(1,14)}=1.81, p=$ 0.20 ; Figure $3 A$ ). To examine refractoriness of the $\mathrm{SO}$-generating networks against stimulation, we analyzed the length of intervals between succeeding SO trains. Subjects $(n=6)$ with $<10$ trains with three or four SO cycles were discarded from this analysis to increase stability of the results. The pause after a stimulation
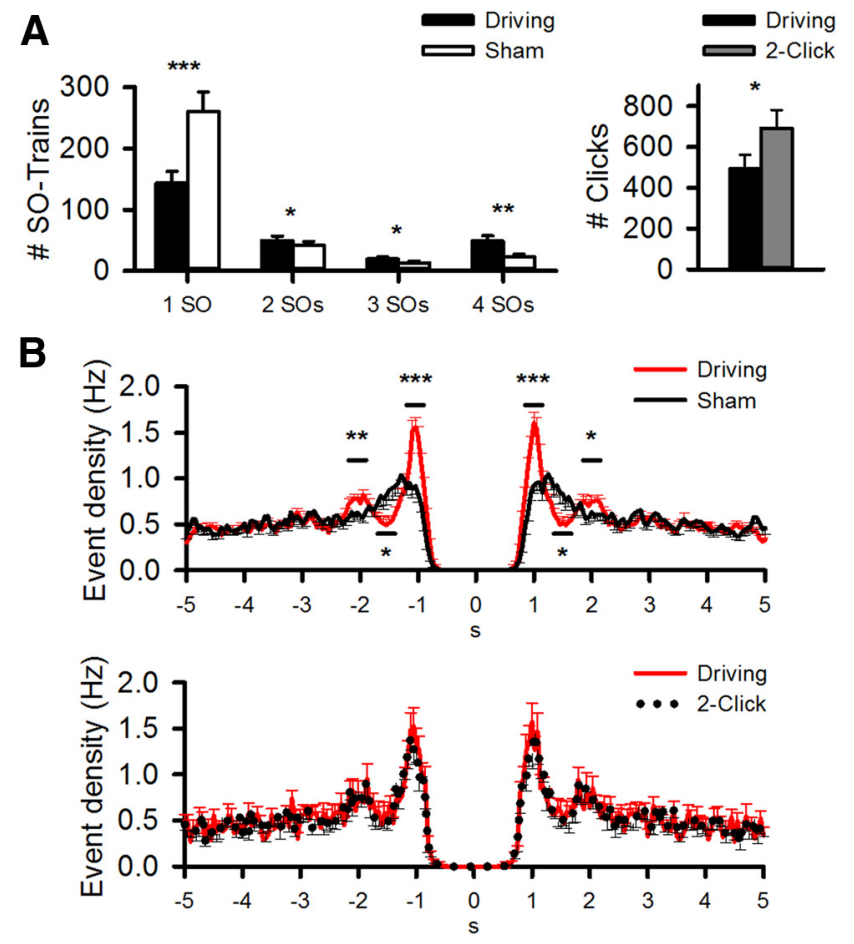

Figure 2. Number of identified SOs and presented clicks in trains. $\boldsymbol{A}$, Mean number ( \pm SEM) of SO trains of one, two, three, or four SO cycles in Study I (Driving stimulation vs Sham; left) and clicks presented in Study II (Driving vs 2-Click; right) during SWS within the 210 min stimulation interval. Compared with Sham stimulation, Driving stimulation prolonged $\mathrm{SO}$ trains as indicated by reduced numbers of single $S O$ cycles and parallel increased amount of trains consisting of two, three, or four SO cycles $\left(F_{(1,14)}=6.548, p=0.023\right.$ and $F_{(3,42)}=26.367, p<0.001$ for Stimulation main effect and Stimulation $\times$ Train length interaction in Study I). $\boldsymbol{B}$, Mean ( \pm SEM) auto-event correlations across all subjects determined from off-line-detected SO events for Study I (top), Driving stimulation (red line) versus Sham stimulation (black line) and for Study II (bottom), Driving stimulation (red line) versus 2-Click stimulation (dotted black line). ${ }^{*} p<0.05,{ }^{* *} p<0.01,{ }^{* * *} p<0.001$, for comparisons between conditions.

sequence (until detection of the next spontaneous negative SO half-wave) was longer during Driving stimulation than during Sham stimulation $\left(F_{(1,9)}=32.56, p<0.001\right)$ with this effect depending on the train length $\left(F_{(3,27)}=4.77, p=0.035\right.$, for Stimulation $\times$ Train length). Pairwise comparison between stimulation conditions revealed that only the pauses after trains consisting of more than one SO cycle were prolonged in comparison with the Sham condition ( $p<0.035$; Fig. $3 B)$.

Comparing the effects of Driving stimulation specifically for trains of two clicks with 2-Click stimulation in Study II revealed that Driving stimulation was associated with a higher SO amplitude in response to the first click $(-106.2 \pm 6.4 \mathrm{vs}-46.6 \pm 5.4$ $\left.\mu \mathrm{V}, F_{(1,12)}=85.611, p<0.001\right)$, but with lower SO amplitude in response to the second click $(-16.1 \pm 2.7$ vs $-32.1 \pm 4.6 \mu \mathrm{V}$, $F_{(1,12)}=10.563, p=0.005$; Fig. $\left.3 C\right)$. Pauses after stimulation did not significantly differ $(10.08 \pm 0.42$ vs $9.59 \pm 0.31 \mathrm{~s}, p=0.121)$. Interestingly, examination of pauses within the Driving stimulation conditions in both Study I and II revealed that intervals following a solitary SO were significantly shorter than intervals following trains with two, three, or four SO cycles (Study I: $p<$ 0.025; Study II: $p<0.031$, for respective pairwise comparisons), whereas intervals following trains with two, three, or four SO cycles did not differ $(p>0.298)$. Collectively, the pattern of pauses observed in Study I and II suggests emergent refractoriness of the SO-generating network against repeated stimulation. It appears that already two succeeding clicks presented in synchrony 
with the endogenous SO rhythm pushes the network into full refractoriness.

\section{Induced fast spindle activity}

Averaging rms fast $(12-15 \mathrm{~Hz})$ spindle activity time-locked to the first clicks of trains during Driving stimulation revealed a pronounced, highly significant increase in fast spindle activity that occurred in phase with the succeeding SO up state (Fig. 4A). Importantly, this phaselocked increase in spindle activity was clearly restricted to the first stimulus presentation independent of the number of clicks delivered in the train $\left(F_{(1,14)}>\right.$ $10.269, p<0.006)$. Succeeding clicks, i.e., presentation of the second, third, or fourth click, remained entirely ineffective $\left(F_{(1,14)}<1.184, p>0.295\right)$. The increase in spindle activity to the first click showed a widespread centroparietal topography, which was virtually identical for the SO trains of different length $\left(F_{(18,252)}>6.550\right.$, $p<0.001$, for Stimulation $\times$ Topography; Fig. $4 B$ for SO trains with three SO cycles).

In Study II, basically the same pattern of a strong increase in spindle activity only to the first but not second click was obtained with the 2-Click stimulation proto$\mathrm{col}$ (response to first vs second click, $F_{(1,18)}$ $=36.01, p<0.001$; Fig. $4 C)$, corroborating the view that induced spindle activity is highly prone to refractoriness. Comparing the effects of Driving stimulation specifically for trains with two clicks and the 2-Click stimulation revealed that Driving stimulation induced a stronger spindle response to the first click $\left(F_{(1,12)}=16.420, p=\right.$ $0.002)$, but a distinctly reduced response to the second $\operatorname{click}\left(F_{(1,12)}=10.423, p=0.007\right.$; Fig. $4 C$ ). Overall, induced spindle activity derived from both clicks was comparable between the conditions $\left(F_{(1,12)}=1.177, p=\right.$ 0.299).

\section{Sleep and spectral power}

In both Study I and II, there were no differences between stimulation conditions in sleep architecture, TST, sleep onset, percentages of time awake, or in non-REM and REM sleep stages for the 210 min stimulation period (all $p s>0.288$; Table 1 ) or for the full nights (all $p s>0.1$ ). In Study I, as expected, analysis of spectral power during SWS indicated that SO peak power was significantly increased during Driving stimulation compared with the Sham control $\left(116.9 \pm 14.2\right.$ vs $96.8 \pm 11.4 \mu \mathrm{V}^{2}, F_{(1,14)}=$ $6.49, p=0.023$; Fig. $5 A$ ), with this increase most pronounced over frontocentral cortical areas $\left(F_{(18,252)}=5.35, p=0.011\right.$, for Stimulation $\times$ Topography). In parallel, during Driving stimulation the peak frequency in the SO range was slightly shifted toward faster frequencies $(0.78 \pm 0.02$ vs $0.72 \pm 0.02 \mathrm{~Hz}$, averaged over all channels; $F_{(1,14)}=14.090, p=0.002$; Fig. $\left.5 B\right)$. There was no difference in spindle power $(12-15 \mathrm{~Hz})$ between the Driving and Sham stimulation conditions $\left(F_{(1,14)}=0.07, p=0.795\right)$.
B
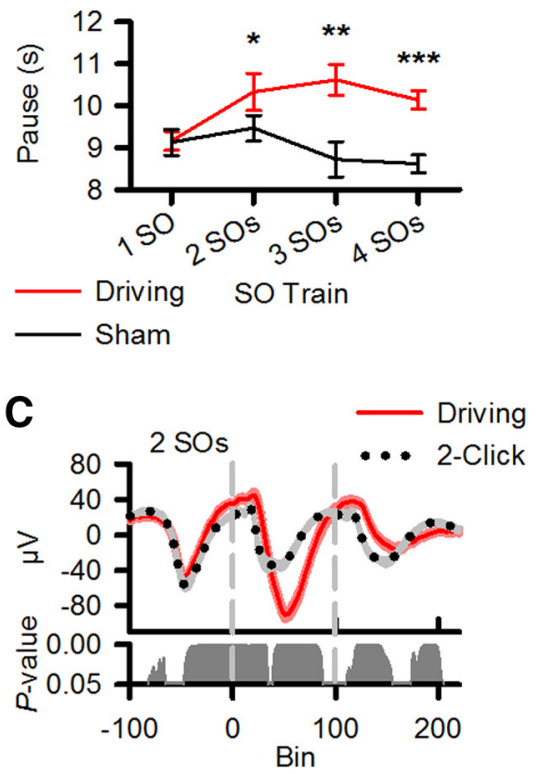

Figure 3. Immediate effects of auditory stimulation. $A$, Mean ( \pm SEM) EEG signal at $C z$ for Driving stimulation (red lines) and Sham condition (black lines) of Study l, time-locked to the presentation of the first click $(0 ; n=15)$. Stimulations are categorized according to the number of successively identified SOs (1, 2, 3, and 4 SOs). Bottom of each part indicates significant differences between conditions. Vertical dashed lines indicate time points of click presentation. Note, intervals between successive clicks were tudes, $\boldsymbol{B}$, Mean ( \pm SEM) interval between SO trains categorized according to the number of SO cycles present in the preceding SO ( 2-Click stimulation, SO amplitude during Driving stimulation is higher following the first click but lower following the first click, specifically in trains comprising two SO cycles, which might be linked to refractoriness in the SO-generating networks, i.e., with Driving stimulation initial clicks occurring at higher depolarization inducing a stronger initial SO response that might subsequently lead to a faster ceasing of the SO train. Note, 100 bins on the $x$-axis correspond on average to $973.0 \pm 11.7 \mathrm{~ms}$.

In Study II, effects of Driving and 2-Click stimulation were quite comparable and there were no significant differences in EEG power and frequency measures of interest between conditions $\left(F_{(1,12)}<0.189, p>0.297\right)$.

Memory performance, controls of the numbers of presented clicks, and behavioral control measures

Study I indicated that Driving stimulation enhanced retention of word pairs across sleep, compared with the Sham condition (difference in recalled word pairs after sleep minus performance at learning before sleep: $24.63 \pm 1.74$ vs $20.25 \pm 1.47$ word pairs, $p=0.024$; Fig. 5C). Overnight retention did not differ between the Driving and 2-Click stimulation condition of Study II $(19.43 \pm 1.40$ vs $21.21 \pm 1.20$ word pairs, $p=0.180)$. In both 

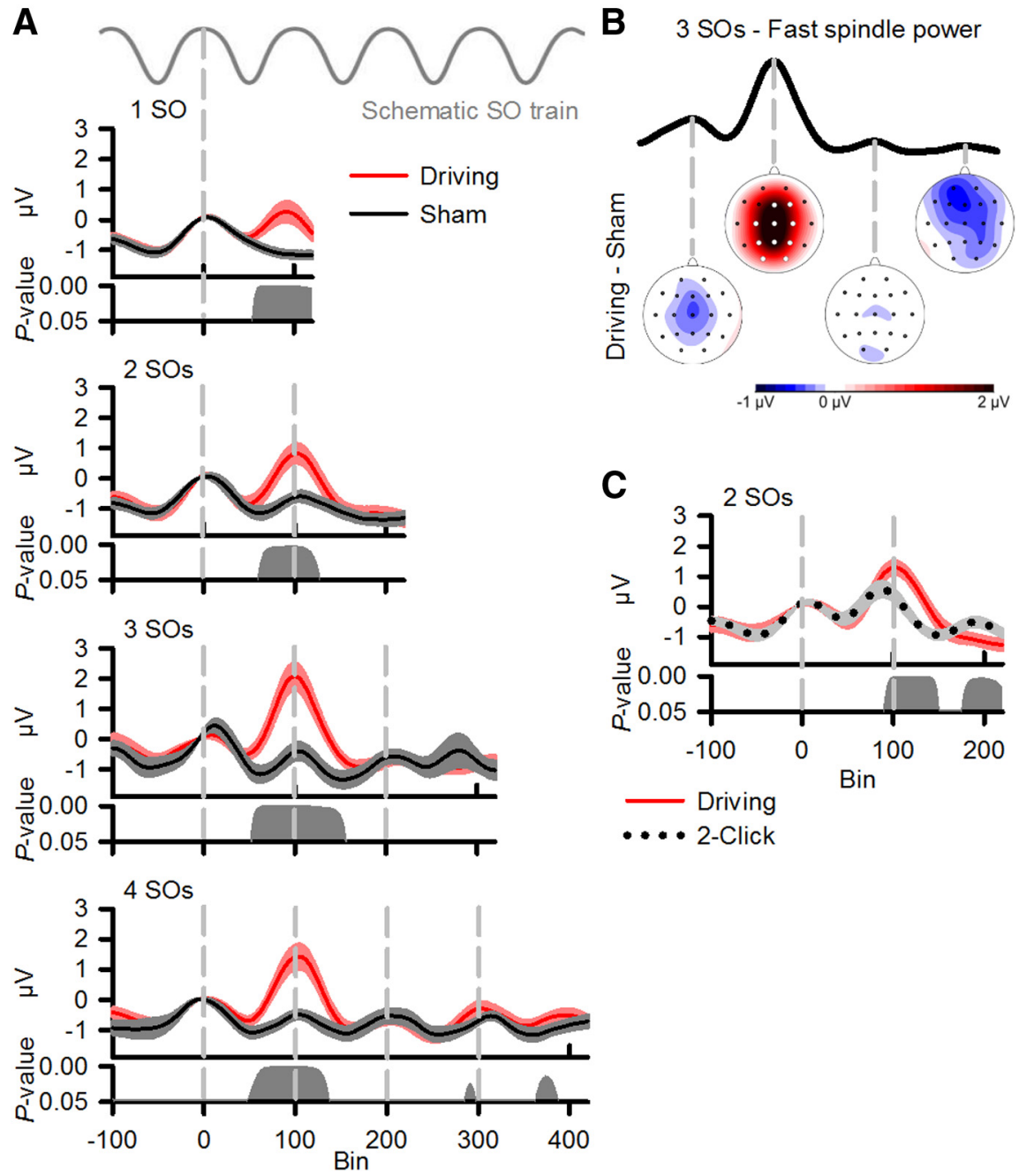

Figure 4. Immediate effects of stimulation on fast spindle responses. $A$, Mean ( \pm SEM) fast spindle activity $(12-15 \mathrm{~Hz}$, rms EEG signal) at ( $\mathrm{Z}$ for Driving stimulation (red lines) and Sham condition (black lines) of Study I, time-locked to the presentation of the first click $(0 ; n=15)$. Stimulations are categorized according to the number of successively identified SOs $(1,2,3$, and 4 SOs). Bottom of each part indicates significant differences between conditions. Vertical dashed lines indicate time points of click presentation. The gray line on top illustrates an S0 train. Note, intervals between successive clicks were standardized to 100 bins to account for the temporal jitter between succeeding $\mathrm{SO}$ s. Driving stimulation profoundly enhances the spindle response only to the first click (with this response peaking around the occurrence of the second click in trains with $>25 \mathrm{~S}$ s), whereas later clicks in a train remain almost entirely ineffective. $\boldsymbol{B}$, Topographical distribution of fast spindle power (12-15 Hz). Difference maps are shown between Driving Stimulation and Sham condition, exemplified for the case of trains with three $\mathrm{SO}$ cycles. Black line on top indicates averaged rms spindle activity for the corresponding train and the vertical lines mark time points of click presentation. Significant ( $p<0.05$, corrected for multiple comparisons) differences between Driving stimulation and Sham condition at specific electrode locations are indicated by filled white circles. C, Corresponding mean ( \pm SEM) fast spindle activity for Study II ( $n=13$ ) comparing effects of the Driving stimulation specifically for trains with two successive clicks (2 SOs, red line) with the 2-Click stimulation (dotted black line). Compared with the 2-Click stimulation, Driving stimulation increases spindle responses to the first click, but reduces spindle responses to the second click. Note, 100 bins on the $x$-axis correspond on average to $973.0 \pm 11.7 \mathrm{~ms}$.

studies, learning performance before sleep was comparable between conditions $(p>0.255)$.

The overall number of presented clicks was significantly greater in the Driving stimulation than 2-Click stimulation condition, which might have confounded memory performance. However, the number of clicks presented did not correlate with the overnight improvement in word recall (Driving stimulation: $r=-0.18$, $p=0.54$; 2-Click stimulation: $r=0.25, p=0.39$ ). Also, we repeated our analyses on a sample including only subjects with comparable numbers of clicks in both stimulation conditions. For this purpose subjects with extremely different numbers of presented clicks in both conditions were removed $(n=2)$, and five additional subjects were run whereby the overall rate of clicks was controlled such that it was comparable or slightly lower in the Driving than 2-Click stimulation condition. Analysis of this regrouped sample $(n=16)$ confirmed that overall click rates were comparable between Driving (428.7 \pm 57.4) and 2-Click stimulation (474.5 \pm 82.0, $p=0.51$ ), while all other results regarding EEG and behavioral measures remained essentially the same as in the original group. Specifically, word-pair recall was on average almost identical in both conditions (Driving: $21.76 \pm 2.05$ word pairs; 2-Click: $21.29 \pm 0.99$ word pairs, $p=0.780$ ). In light of these analyses a substantial influence of the overall number of presented clicks on sleepassociated consolidation processes can be excluded.

In both studies, no significant differences were found for performance on the Psychomotor Vigilance Test at learning before sleep (Driving vs Sham: $332.88 \pm$ 6.65 vs $324.45 \pm 7.13 \mathrm{~ms}$; Driving vs 2-Click: $277.37 \pm 12.14$ vs $283.23 \pm 12.16$ $\mathrm{ms}$ ) or at retrieval after sleep (Driving vs Sham: $333.62 \pm 8.32$ vs $333.96 \pm 11.07$ ms; Driving vs 2-Click: $276.05 \pm 8.34$ vs $276.93 \pm 10.20 \mathrm{~ms}$, for all $p>0.201$ ). Stimulation did also not affect control measures of executive function (for all $p s>0.88)$. Likewise, measures of subjective sleepiness and mood did not reveal any differences between conditions $(p>$ $0.39)$.

\section{Discussion}

Our data indicate that repetitive, closedloop auditory stimulation of SOs during SWS as established with the Driving stimulation protocol effectively prolongs SO trains, enhances SO amplitudes together with spindle activity phase-locked to the SO up state, and distinctly improves the overnight retention of declarative wordpair memories, if compared with a Sham condition devoid of any stimulation. However, the Driving stimulation protocol, which was basically designed to drive trains of multiple succeeding SO cycles by presenting up to four clicks in succession, did not prove superior to a less driving "2-Click" stimulation procedure adopted from a previous study (Ngo et al., 2013b), where just two clicks were given in succession. There was no difference between the stimulation protocols in the efficacy to prolong SO trains or enhance SO amplitudes, nor were there differences in induced spindle activity or memory performance. The failure to enhance efficacy of closed-loop stimulation by enhancing the repetitions of stimulation in an SO train indicates the presence of mechanisms that prevent an overdriving of SO activity. 
Table 1. Sleep during the $\mathbf{2 1 0}$ min stimulation period and the whole night

\begin{tabular}{|c|c|c|c|c|c|c|}
\hline \multirow[b]{2}{*}{ Parameter } & \multicolumn{3}{|l|}{ Study I } & \multicolumn{3}{|l|}{ Study II } \\
\hline & Driving & Sham & $P$ value & Driving & 2-Click & $P$ value \\
\hline \multicolumn{7}{|c|}{210 in stimulation interval } \\
\hline W (\%) & $1.3 \pm 0.6$ & $2.0 \pm 1.1$ & 0.620 & $2.3 \pm 0.9$ & $1.1 \pm 0.9$ & 0.288 \\
\hline S1 (\%) & $4.2 \pm 1.7$ & $3.8 \pm 1.0$ & 0.874 & $5.8 \pm 0.8$ & $5.0 \pm 0.6$ & 0.309 \\
\hline S2 (\%) & $57.2 \pm 3.5$ & $57.8 \pm 3.6$ & 0.779 & $50.2 \pm 2.1$ & $51.8 \pm 2.4$ & 0.540 \\
\hline SWS (\%) & $27.5 \pm 3.8$ & $27.7 \pm 3.1$ & 0.960 & $28.5 \pm 2.3$ & $31.0 \pm 2.7$ & 0.369 \\
\hline REM (\%) & $9.8 \pm 2.8$ & $8.7 \pm 1.5$ & 0.666 & $13.2 \pm 1.7$ & $11.1 \pm 1.2$ & 0.312 \\
\hline \multicolumn{7}{|l|}{ Whole night } \\
\hline TST (min) & $410.0 \pm 6.1$ & $421.7 \pm 4.4$ & 0.100 & $417.8 \pm 3.9$ & $422.7 \pm 2.5$ & 0.284 \\
\hline W (\%) & $2.1 \pm 0.7$ & $4.2 \pm 1.9$ & 0.218 & $5.4 \pm 1.9$ & $2.8 \pm 1.3$ & 0.103 \\
\hline S1 (\%) & $6.1 \pm 1.0$ & $5.4 \pm 0.5$ & 0.606 & $6.2 \pm 0.7$ & $5.8 \pm 0.9$ & 0.725 \\
\hline S2 (\%) & $57.6 \pm 1.8$ & $56.3 \pm 2.1$ & 0.458 & $50.1 \pm 2.0$ & $53.0 \pm 1.8$ & 0.210 \\
\hline SWS (\%) & $16.2 \pm 1.4$ & $16.6 \pm 1.4$ & 0.725 & $21.6 \pm 1.0$ & $21.2 \pm 1.8$ & 0.820 \\
\hline REM (\%) & $17.9 \pm 1.3$ & $17.5 \pm 1.2$ & 0.796 & $16.7 \pm 1.6$ & $17.2 \pm 1.2$ & 0.737 \\
\hline
\end{tabular}

Mean ( \pm SEM) percentage of time spent in different sleep stages during the $210 \mathrm{~min}$ stimulation period and $7 \mathrm{~h}$ nocturnal sleep interval for Study I (Driving stimulation vs Sham) and Study II (Driving stimulation vs 2-Click stimulation). There were no significant differences between conditions. TST, total sleep time (in minutes); W, wake; S1, stage 1 sleep; $\$ 2$, stage 2 sleep.

A
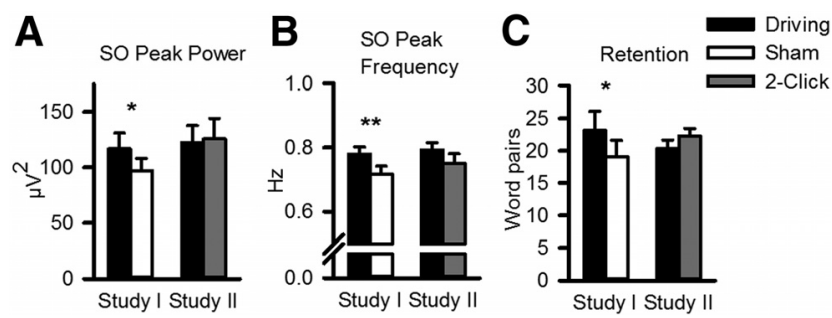

Figure 5. Auditory stimulation enhances $S 0$ power and memory retention. $\boldsymbol{A}, \boldsymbol{B}$, Mean ( \pm SEM) spectral power of the individually determined SO peak $(\boldsymbol{A})$ and mean ( \pm SEM) SO peak frequency $(\boldsymbol{B})$ during SWS within the 210 min stimulation period averaged across all subjects and EEG electrodes. $\boldsymbol{C}$, Mean ( \pm SEM) retention of word pairs across the $7 \mathrm{~h}$ nocturnal sleep period (expressed as difference in recalled word pairs at retrieval testing after sleep minus performance at an immediate recall test before sleep). Data are presented separately for the Driving stimulation (black bars) and Sham condition (empty bars) of Study I and the Driving stimulation (black bars) and 2-Click stimulation condition (gray bars) of Study II. ${ }^{*} p<0.05$, ${ }^{* *} p<0.01$ for pairwise comparisons between conditions.

\section{More than two clicks in a train does not further enhance SO activity or memory}

The present study adds support to the notion that closed-loop stimulation in general is an effective tool to specifically manipulate oscillatory EEG activity (Berényi et al., 2012; Paz et al., 2013). If applied time-locked to the ongoing SO up states during SWS, the presentation of clicks reliably induced further SOs with high amplitude, accompanied by surges of spindle activity in phase with the invoked SO up state. At the behavioral level the changes express themselves in an enhanced overnight retention of declarative memories, altogether corroborating the view that the induced SOs are functionally effective in the same way as endogenous SOs (Clemens et al., 2007; Chauvette et al., 2012; Cox et al., 2012; Phillips et al., 2012; Ruch et al., 2012; Wilhelm et al., 2013). In fact, the changes following both closed-loop stimulation protocols used here are highly specific, as indicated by comparisons with the effects of a Sham control condition, which were performed for the 2-Click protocol in a previous study (Ngo et al., 2013b) and for the Driving stimulation protocol here. Accordingly, both stimulation protocols left the gross sleep architecture, the amount of SWS, REM sleep, and number of awakenings entirely unaffected and also the changes induced in EEG power during SWS were restricted to the SO frequency band. Thus, general features of sleep remained untouched, but closed- loop SO stimulation chiefly induced an acute temporal restructuring of $\mathrm{SO}$ and nested spindle activity.

Indeed a closer examination of the acute dynamics of induced SO and spindle activity after Driving stimulation and 2-Click stimulation revealed surprising insights into the network conditions underlying the generation of SOs. First, Driving stimulation was indeed capable of inducing SO trains of up to three to four successive cycles. Such trains of several successive SOs have been suspected to be particularly powerful in promoting sleepdependent memory consolidation, as their occurrence is increased after intense learning (Mölle et al., 2011). However, the occurrence of such SO trains is still relatively rare, and even more so under nonstimulated conditions ( $<10 \%$ of all SOs), indicating an overall low tendency for the spontaneous occurrence of longer groups of SOs, and that it is difficult to evoke a resonant response of this length. As to the generation of SO trains, network conditions appear to be inert during SWS. This is further supported by the effects of 2-Click stimulation which, as revealed by auto-event correlations (Fig. 2A), exhibited the same capability to induce longer trains of SO events as the Driving stimulation protocol. Also, overall SO numbers and amplitudes were comparable for the two stimulation protocols, although the 2-Click protocol lacked instances of three or four click presentations in succession. Altogether this picture indicates that $>2$ click stimulations in succession, as they occurred only during Driving stimulation, remain basically ineffective. This conclusion is ultimately supported also by the fact that the memory-enhancing effect of both stimulation protocols was equivalent.

The comparison of acute effects of 2-Click stimulation with those of Driving stimulation resulting in trains of two clicks in succession suggested that Driving stimulation even enhanced the network's inertia to produce SOs, as the decrease in SO amplitude as well as the parallel decrease in induced phase-locked spindle activity across the two induced $\mathrm{SO}$ cycles was much greater for the Driving stimulation protocol than for the 2-Click stimulation protocol. However, the comparison of these two conditions, although apparently alike, may be misleading given that the SO amplitude induced by the first click in the Driving stimulation condition was significantly greater than that to the first click in the 2-Click stimulation protocol. This finding must be ascribed to the fact that unlike the 2-Click protocol where the first click was always followed by a second click, the Driving stimulation protocol provided a second click only when the first click induced a suprathreshold negative SO half-wave. Nevertheless, the view that Driving stimulation aggravated refractoriness in SOgenerating networks is indeed supported by the pattern of pauses until stimulation was resumed. In both Study I and II these pauses during Driving stimulation were longer after the induction of SO trains with two or more SO cycles than after induction of a solitary SO, suggesting that refractoriness builds up already with the second induced SO cycle, whereas pauses after spontaneous trains (during the Sham condition) did not vary as a function of train length. Most important, however, is the fact that all of the pauses following the induction of trains with two or more SO cycles were significantly longer than the pauses after the respective trains of the Sham condition, while pauses after solitary SOs were comparable between the Driving stimulation and Sham condition.

\section{Spindle refractoriness as possible mechanism preventing overdriving SO stimulation}

A key finding of our studies is that independent of the stimulation protocol, only the first click induced a robust increase of fast 
(12-15 Hz) spindle activity phase-locked to the SO up state, whereas increases in spindle activity accompanying the up states of succeeding SO cycles were comparable to those seen during Sham conditions. This finding indicates that spindle-generating networks in thalamic circuitry build up an immediate resistance to stimulation (von Krosigk et al., 1993), which most likely reflects refractoriness of spindle generation. Thalamic spindle generation is well known to underlie refractoriness periods (between 5 and $20 \mathrm{~s}$, in the ferret) due to the persistent activation of the hyperpolarization-activated cation current $I_{\mathrm{h}}$ in thalamocortical cells (Destexhe et al., 1998; Lüthi and McCormick, 1998). The dynamical upregulate of $I_{\mathrm{h}}$ in these networks, which also appears to be partly controlled by descending corticothalamic projections, is considered the critical determinant of the time course of the refractory period (Bonjean et al., 2011). Importantly, the thalamocortical cells confer relative refractoriness to the entire thalamocortical network, including cortical networks generating regular SO activity as well as pathological spike-and-wave seizure activity (Destexhe et al., 1998).

One might argue that, rather than refractoriness, the profound decrease in evoked fast spindle activity with the occurrence of the second and further clicks represents adaptation or habituation favored by a regular and predictable timing of the stimuli. This is unlikely because the decrease in evoked spindle activity emerged rather abruptly with the second click, whereas habituation would be expected to produce a more gradual decrease with increasing numbers of successive clicks. It is also important to note that, as to its temporal features, the Driving stimulation protocol resembles a randomized stimulation, because click presentation is coupled to the detection of an SO-negative half-wave, and the SO rhythm per se exhibits substantial temporal jitter. We additionally analyzed data taken from a previous study (Ngo et al., 2013a) in which sleeping subjects were presented with randomly (vs regularly) occurring clicks (at an average rate of 0.8 $\mathrm{Hz}$ ). Fast spindle rms responses to clicks occurring after short ISIs $(0.125-0.5 \mathrm{~s})$ were distinctly lower than to clicks occurring after a long ISI ( $2-5 \mathrm{~s}, p=0.015)$. In fact, there was virtually no remaining fast spindle response to the clicks after short ISIs when the portion of the response caused by the preceding click was removed. Altogether, this picture corroborates the view that refractoriness rather than habituation determined the temporal dynamics of fast spindle activity in response to Driving stimulation.

Interestingly, also in spontaneous SO trains spindle activity associated with the first SO cycle is of particular importance (Mölle et al., 2011). Fast (12-15 Hz) spindle activity is highest during the up state of the first SO cycle and lowest during the last cycle of a train. Moreover, intense declarative learning before sleep does not only enhance the number of SOs occurring in a train but also enhanced spindle activity, most profoundly during up states of SOs initiating an SO train, as well as shortly before an identifiable SO. The present findings in conjunction with those previous analyses speak for a loop-like scenario where spindles, by promoting excitability changes in local cortical pyramidal networks (Destexhe et al., 2007; Ayoub et al., 2013), enhance the likelihood and amplitude of succeeding SO cycles. Emergent depolarization in the succeeding $\mathrm{SO}$ cycle, in turn, exerts a driving influence on thalamic spindle generation (Contreras and Steriade, 1995). However, due to refractoriness in thalamocortical neurons the resulting increase in spindle activity is profoundly decreased (Mölle et al., 2011). Basically, this view assumes an initiating role of spindles for trains of $\mathrm{SO}$, which is also in accord with the occurrence of spindles in the absence of strong SO ac- tivity during human non-REM sleep stage 2, typically preceding SWS periods with consolidated SO activity (for review, see Kim et al., 2012). Indeed, results from the Driving stimulation protocol of the present study indicate that closed-loop click stimulation can produce a robust increase in spindle activity also when concomitantly induced SO activity is marginal, i.e., remains below the criterion threshold used here for identifying SOs. This actually happened in all instances when the on-line detection of a negative SO half-wave peak was followed by the presentation of only a single click (Fig. $3 A$ ). Considering also that such single-click events represent a substantial portion of SO events during Driving stimulation, it is tempting to speculate that the closed-loop auditory stimulation of SOs is at least partly effective via a primary influence on spindle-generating networks. If so, this raises the question, to be answered in future studies, whether the closed-loop presentation of single, isolated auditory stimuli already provides the maximum effect with regard to the stimulation of SO trains.

Our studies confirm the efficacy of closed-loop auditory stimulation presented in phase with SO up states, to prolong SO trains, to enhance SO amplitude and SO up state-associated spindle activity, and to eventually improve retention of declarative word-pair memories (Ngo et al., 2013b). Importantly, compared with the stimulation of two successive SO cycles, the repetitive stimulation of more than two cycles in succession does not increase efficacy of stimulation. This apparent insensitivity of the network to the enhancing effect of Driving stimulation (comprising more than two successive SO cycles) indicates the presence of a protective mechanism preventing the thalamocortical system from going into hypersynchrony in conditions of overdriving SO activity. The mechanism appears to be linked to the refractoriness of SO up state-associated spindle activity, because this was enhanced only after the initial click stimulus. Thus, efficacy of the Driving stimulation protocol not exceeding that of the 2-Click protocol reveals a protective counter mechanism, which basically limits the effects of SO stimulation, and whose impairment might contribute to seizure activity in pathological conditions.

\section{References}

Achermann P, Borbély AA (1997) Low-frequency ( $<1 \mathrm{~Hz})$ oscillations in the human sleep electroencephalogram. Neuroscience 81:213-222. CrossRef Medline

Ayoub A, Aumann D, Hörschelmann A, Kouchekmanesch A, Paul P, Born J, Marshall L (2013) Differential effects on fast and slow spindle activity, and the sleep slow oscillation in humans with carbamazepine and flunarizine to antagonize voltage-dependent $\mathrm{Na}+$ and $\mathrm{Ca}^{2+}$ channel activity. Sleep 36:905-911. CrossRef Medline

Bazhenov M, Timofeev I, Steriade M, Sejnowski TJ (2002) Model of thalamocortical slow-wave sleep oscillations and transitions to activated States. J Neurosci 22:8691-8704. Medline

Berényi A, Belluscio M, Mao D, Buzsáki G (2012) Closed-loop control of epilepsy by transcranial electrical stimulation. Science 337:735-737. CrossRef Medline

Bergmann TO, Mölle M, Diedrichs J, Born J, Siebner HR (2012) Sleep spindlerelated reactivation of category-specific cortical regions after learning facescene associations. Neuroimage 59:2733-2742. CrossRef Medline

Bonjean M, Baker T, Lemieux M, Timofeev I, Sejnowski T, Bazhenov M (2011) Corticothalamic feedback controls sleep spindle duration in vivo. J Neurosci 31:9124-9134. CrossRef Medline

Chauvette S, Seigneur J, Timofeev I (2012) Sleep oscillations in the thalamocortical system induce long-term neuronal plasticity. Neuron 75:11051113. CrossRef Medline

Chen JY, Chauvette S, Skorheim S, Timofeev I, Bazhenov M (2012) Interneuron-mediated inhibition synchronizes neuronal activity during slow oscillation. J Physiol 590:3987-4010. CrossRef Medline

Clemens Z, Mölle M, Eross L, Barsi P, Halász P, Born J (2007) Temporal coupling of parahippocampal ripples, sleep spindles and slow oscillations in humans. Brain 130:2868-2878. CrossRef Medline 
Contreras D, Steriade M (1995) Cellular basis of EEG slow rhythms: a study of dynamic corticothalamic relationships. J Neurosci 15:604-622. Medline

Cox R, Hofman WF, Talamini LM (2012) Involvement of spindles in memory consolidation is slow wave sleep-specific. Learn Mem 19:264-267. CrossRef Medline

Cox R, Korjoukov I, de Boer M, Talamini LM (2014) Sound asleep: processing and retention of slow oscillation phase-targeted stimuli. PLoS One 9:e101567. CrossRef Medline

de Guzman PH, Nazer F, Dickson CT (2010) Short-duration epileptic discharges show a distinct phase preference during ongoing hippocampal slow oscillations. J Neurophysiol 104:2194-2202. CrossRef Medline

Destexhe A, Contreras D, Steriade M (1998) Mechanisms underlying the synchronizing action of corticothalamic feedback through inhibition of thalamic relay cells. J Neurophysiol 79:999-1016. Medline

Destexhe A, Hughes SW, Rudolph M, Crunelli V (2007) Are corticothalamic 'up' states fragments of wakefulness? Trends Neurosci 30:334-342. CrossRef Medline

Huber R, Ghilardi MF, Massimini M, Tononi G (2004) Local sleep and learning. Nature 430:78-81. CrossRef Medline

Kim A, Latchoumane C, Lee S, Kim GB, Cheong E, Augustine GJ, Shin HS (2012) Optogenetically induced sleep spindle rhythms alter sleep architectures in mice. Proc Natl Acad Sci U S A 109:20673-20678. CrossRef Medline

Lüthi A, McCormick DA (1998) Periodicity of thalamic synchronized oscillations: the role of $\mathrm{Ca}^{2+}$-mediated upregulation of Ih. Neuron 20:553563. CrossRef Medline

Marshall L, Mölle M, Hallschmid M, Born J (2004) Transcranial direct current stimulation during sleep improves declarative memory. J Neurosci 24:9985-9992. CrossRef Medline

Marshall L, Helgadóttir H, Mölle M, Born J (2006) Boosting slow oscillations during sleep potentiates memory. Nature 444:610-613. CrossRef Medline

Massimini M, Ferrarelli F, Esser SK, Riedner BA, Huber R, Murphy M, Peterson MJ, Tononi G (2007) Triggering sleep slow waves by transcranial magnetic stimulation. Proc Natl Acad Sci U S A 104:8496-8501. CrossRef Medline

Mölle M, Marshall L, Gais S, Born J (2002) Grouping of spindle activity during slow oscillations in human non-rapid eye movement sleep. J Neurosci 22:10941-10947. Medline

Mölle M, Bergmann TO, Marshall L, Born J (2011) Fast and slow spindles during the sleep slow oscillation: disparate coalescence and engagement in memory processing. Sleep 34:1411-1421. CrossRef Medline

Nazer F, Dickson CT (2009) Slow oscillation state facilitates epileptiform events in the hippocampus. J Neurophysiol 102:1880-1889. CrossRef Medline

Ngo HV, Claussen JC, Born J, Mölle M (2013a) Induction of slow oscillations by rhythmic acoustic stimulation. J Sleep Res 22:22-31. CrossRef Medline
Ngo HV, Martinetz T, Born J, Mölle M (2013b) Auditory closed-loop stimulation of the sleep slow oscillation enhances memory. Neuron 78:545553. CrossRef Medline

Olcese U, Esser SK, Tononi G (2010) Sleep and synaptic renormalization: a computational study. J Neurophysiol 104:3476-3493. CrossRef Medline

Paz JT, Davidson TJ, Frechette ES, Delord B, Parada I, Peng K, Deisseroth K, Huguenard JR (2013) Closed-loop optogenetic control of thalamus as a tool for interrupting seizures after cortical injury. Nat Neurosci 16:64-70. CrossRef Medline

Phillips KG, Bartsch U, McCarthy AP, Edgar DM, Tricklebank MD, Wafford KA, Jones MW (2012) Decoupling of sleep-dependent cortical and hippocampal interactions in a neurodevelopmental model of schizophrenia. Neuron 76:526-533. CrossRef Medline

Plihal W, Born J (1997) Effects of early and late nocturnal sleep on declarative and procedural memory. J Cogn Neurosci 9:534-547. CrossRef Medline

Rechtschaffen A, Kales A (1968) A manual of standardized terminology, techniques and scoring system for sleep stages of human subjects. Washington DC: US Department of Health, Education and Welfare Public Health Service.

Ruch S, Markes O, Duss SB, Oppliger D, Reber TP, Koenig T, Mathis J, Roth C, Henke K (2012) Sleep stage II contributes to the consolidation of declarative memories. Neuropsychologia 50:2389-2396. CrossRef Medline

Seibt J, Dumoulin MC, Aton SJ, Coleman T, Watson A, Naidoo N, Frank MG (2012) Protein synthesis during sleep consolidates cortical plasticity in vivo. Curr Biol 22:676-682. CrossRef Medline

Siapas AG, Wilson MA (1998) Coordinated interactions between hippocampal ripples and cortical spindles during slow-wave sleep. Neuron 21:1123-1128. CrossRef Medline

Steriade M (2003) The corticothalamic system in sleep. Front Biosci 8:d878-d899. CrossRef Medline

Timofeev I (2011) Neuronal plasticity and thalamocortical sleep and waking oscillations. Prog Brain Res 193:121-144. CrossRef Medline

Tononi G, Cirelli C (2014) Sleep and the price of plasticity: from synaptic and cellular homeostasis to memory consolidation and integration. Neuron 81:12-34. CrossRef Medline

von Krosigk M, Bal T, McCormick DA (1993) Cellular mechanisms of a synchronized oscillation in the thalamus. Science 261:361-364. CrossRef Medline

Wilhelm I, Rose M, Imhof KI, Rasch B, Büchel C, Born J (2013) The sleeping child outplays the adult's capacity to convert implicit into explicit knowledge. Nat Neurosci 16:391-393. CrossRef Medline

Yang G, Lai CS, Cichon J, Ma L, Li W, Gan WB (2014) Sleep promotes branch-specific formation of dendritic spines after learning. Science 344: 1173-1178. CrossRef Medline 